\title{
Ce qu'Internet fait à la diffusion des croyances
}

\section{Gérald Bronner}

\section{OpenEdition}

Journals

Édition électronique

URL : http://journals.openedition.org/ress/805

DOI : $10.4000 /$ ress.805

ISSN : 1663-4446

\section{Éditeur}

Librairie Droz

\section{Édition imprimée}

Date de publication : 15 octobre 2011

Pagination : $35-60$

ISBN : 978-2-600-01551-6

ISSN : 0048-8046

Référence électronique

Gérald Bronner, "Ce qu'Internet fait à la diffusion des croyances », Revue européenne des sciences sociales [En ligne], 49-1 | 2011, mis en ligne le 01 janvier 2015, consulté le 10 décembre 2020. URL http://journals.openedition.org/ress/805 ; DOI : https://doi.org/10.4000/ress.805 


\title{
CE QU'INTERNET FAIT \\ À LA DIFFUSION DES CROYANCES
}

GÉRALD BRONNER

CNRS / Université de Strasbourg gerald.bronner@misha.fr

Résumé. Internet suscite beaucoup d'espoirs et de craintes. Certains prétendent que cet outil sera favorable à l'émergence de sociétés de la connaissance à condition de réduire préalablement la fracture numérique et cognitive. Cet article prend prétexte de cette idée pour évaluer quels sont les réels rapports de force qui se jouent entre croyances et connaissances sur la toile. II montre, d'une part, que l'amplification de la diffusion de l'information est favorable à l'expression du biais de confirmation qui est un des mécanismes fondamentaux de la pérennité des croyances. II souligne, d'autre part, qu'Internet est un marché cognitif très sensible à la structuration de l'offre et donc à la motivation des offreurs, ce qui confère un avantage décisif à l'empire des croyances contre celui de la connaissance. Ce dernier point est mesuré dans cet article de façon quantitative sur des thèmes de croyances diverses.

Mots-clés: croyances; société de la connaissance; information;Internet; marché cognitif

\begin{abstract}
The Internet is the source of many hopes and fears. Some claim that this tool will help lead to the emergence of knowledge societies if digital and cognitive gaps can be reduced beforehand. This article takes that idea as a basis to then evaluate what are the real power relations played out between beliefs and knowledge on the web. It shows that the amplification of the dissemination of information is conducive to the expression of "confirmation bias" which is one of the mechanisms which permit beliefs to survive. It also stresses that Internet is a cognitive market which is very sensitive to structuring of supply and therefore the motivation of suppliers, which confers a decisive advantage to the "empire of beliefs". This last point is measured quantitatively in this paper on various topics of belief.
\end{abstract}

Keywords: beliefs; knowledge; societies; information; Internet; cognitive market 
INTRODUCTION

L'utopie de la société de la connaissance et l'empire des croyances

Comme de nombreuses innovations technologiques majeures, Internet a suscité bien des craintes et des espoirs, plusieurs livres qui ont rencontré un certain succès de librairie attestent des unes comme des autres'. Gaschke (2009) a sans doute raison de supposer que ces sentiments - elle évoque surtout les espoirs - ont quelque chose d'idéologique, mais je laisserai de côté sa thèse selon laquelle cette idéologie serait celle, polymorphe, du capitalisme qui trouverait là une forme de transcendance en promettant l'accès au savoir pour tous. Je ne ferai que constater que le Web, parce qu'il permet une massification exponentielle de la diffusion de l'information et potentiellement une accessibilité sans coût à cette information pour tous, a paru, à certains, pouvoir donner corps au programme de la société de la connaissance. Cette notion, qui a été utilisée pour la première fois par Drucker (1969) et a de nombreuses fois été reprise et approfondie ${ }^{2}$, se fonde sur une constatation: les mutations de nos systèmes de productions ont conduit nos sociétés à considérer le savoir et l'innovation comme les facteurs clés du développement économique et l'accessibilité pour tous à ce savoir comme l'enjeu fondamental de notre avenir démocratique. La théorie de la société de la connaissance se veut à la fois analyse et projet politique. De ce point de vue, le rapport mondial de l'UNESCO : Vers les sociétés du savoir, qui reprend cette notion à son compte et milite en faveur des sociétés de la connaissance, peut être considéré comme une sorte de manifeste (p. i7) :

À présent, la diffusion des nouvelles technologies et l'avènement de l'Internet comme réseau public paraissent ouvrir de nouvelles chances pour élargir cet espace public du savoir. Serions-nous désormais dotés des moyens qui permettraient un accès égal et universel à la connaissance, et un authentique partage? Celui-ci devrait alors être la clé de voûte de véritables sociétés du savoir qui soient source d'un développement humain et durable.

I Voir par exemple Tapscott (2008) pour les espoirs et Bauerlein (2008) pour les craintes.

2 Par exemple Mansell Wehn (1998) ou Stehr (1994). 
Ce rapport se fonde essentiellement sur une analyse en deux points, il constate, d'une part, l'existence d'une fracture numérique, c'est-à-dire l'inégalité d'accès aux sources, aux contenus et aux infrastructures de l'information entre les sociétés et à l'intérieur même de ces sociétés. Cette fracture numérique empêcherait l'essor des sociétés du savoir. Il serait donc urgent, non seulement pour servir les valeurs démocratiques, mais encore pour favoriser partout l'essor économique, de réduire cette «fracture numérique».

D’autre part, précise ce rapport, cet effort serait nécessaire mais pas suffisant car: «La transformation d'une information en savoir suppose un travail de réflexion. En tant que telle, une information n'est qu'une donnée brute, la matière première de l'élaboration d'un savoir » (p. 47). Il faut donc prendre acte de l'existence d'une «fracture cognitive» qui qualifie l'inégalité des individus (essentiellement en raison d'un différentiel de niveau d'éducation) face à «la maîtrise de certaines compétences cognitives, critiques et théoriques, dont le développement est précisément l'objet des sociétés du savoir» (ibid.). Cette maîtrise permettrait à l'individu, selon les auteurs du rapport, de s'orienter dans la mer de l'information pour trouver la terre ferme de la connaissance.

Il n'est pas difficile d'éprouver de la sympathie pour les intentions morales et politiques de ce programme, mais il n'est pas obligatoire d'en accepter sans discuter les analyses. Le diagnostic du rapport de l'Unesco, qui revendique l'expertise de noms prestigieux : Régis Debray, Jacques Derrida, Jean-Pierre Dupuy, Françoise Héritier, Julia Kristeva, Bruno Latour, Jean d’Ormesson, Paul Ricœur, Dan Sperber, Alain Touraine et beaucoup d'autres ${ }^{3}$, paraît discutable si l'on tient compte des mécanismes de concurrence réelle entre les produits de la connaissance et ceux de la croyance ${ }^{4}$. Mon sujet ne sera pas de discuter point par point le programme de la société de la connaissance, mais de m’en servir comme élément introductif parce qu’il révèle, selon moi, une erreur d'appréciation

3 Ces auteurs ne peuvent être comptables des thèses défendues par le rapport, celui-ci les évoque, cependant, en reconnaissant qu'il «a bénéficié des contributions initiales et des analyses» de ces auteurs.

4 Je discuterai plus bas de la ligne de démarcation que l'on peut faire passer entre les deux. 
sur les processus de diffusion non sélective de l'information qui caractérise les rapports entre offre et demande cognitives, en particulier sur Internet.

Le marché cognitif ${ }^{5}$ dans les sociétés occidentales contemporaines est globalement libéral dans la mesure où, à de rares exceptions près, les produits ne subissent pas de taxation ou d'interdiction étatique. Ce libéralisme cognitif est consubstantiel à la constitution même des démocraties, mais il est aussi rendu possible par les innovations technologiques. De ce point de vue, Internet, qui suscite tant d'espoirs de la part des promoteurs des sociétés de la connaissance, en est une manifestation emblématique. Des chercheurs (Autret, 2002) ont ainsi affirmé que l'information produite sur notre planète en cinq ans, à la fin du $\mathrm{Xx}^{\mathrm{e}}$ siècle et au début du $\mathrm{XxI}^{\mathrm{e}}$ siècle, avait été supérieure quantitativement à l'ensemble de l'information imprimée depuis Gutenberg.

\section{L'AMPLIFICATION DU BIAIS DE CONFIRMATION}

Il est devenu banal de rappeler que la légitimité des grands systèmes idéologiques et religieux qui, pendant longtemps, assurèrent une certaine homogénéité représentationnelle dans nos sociétés est largement contestée. Leur légitimité oligopolistique plus qu’affaiblie, le marché a mécaniquement vu apparaître une multitude de propositions parcellisées. Cette balkanisation des représentations dans les sociétés contemporaines permet aux individus de composer, en quelque sorte, «sur mesure » des systèmes de négociation avec le monde. Cette libre composition ne rencontre donc pas toujours l'expression de la pure connaissance. Elle est rendue aisée par la présence plurielle des propositions cognitives sur le marché et par leur plus grande accessibilité. Il est possible aujourd'hui d'adhérer à une représentation du monde tendant vers l'idiosyncrasie et convoquant, par exemple, un peu de christianisme, un peu de bouddhisme, quelques éléments de mythologie conspirationniste tout en croyant que notre santé est gouvernée par des ondes et en revendiquant, cependant, un certain esprit rationaliste. La conséquence la moins visible et pourtant la plus déterminante de cet état de fait est que toutes les conditions sont alors réunies

5 Les limites volumétriques de cet article ne me permettent pas de développer cette notion qui l'est dans Bronner (2003). 
pour que le biais de confirmation puisse donner la pleine mesure de ses capacités à nous détourner de la vérité. De toutes les tentations inférentielles qui pèsent sur la logique ordinaire, le biais de confirmation est sans doute celui qui est le plus déterminant dans les processus qui pérennisent les croyances. On en trouve une description sous la plume de Bacon dans l'aphorisme 46 du Novum Organum :

C'est une erreur constante et propre à l'entendement humain d'être mis en branle davantage par les affirmatives que par les négatives, alors que, en bonne règle, il devrait se prêter également aux deux. Tout au contraire, lorsqu'il faut établir un axiome vrai, la force de l'instance négative est plus grande.

On peut dire que, de ce point de vue, Bacon anticipe les résultats des expérimentations menées par Wason en 1966 sur le biais de confirmation. Celle-ci consistait à proposer à des sujets volontaires un jeu, en apparence assez simple, impliquant quatre cartes.

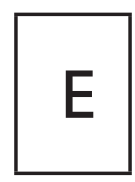

।

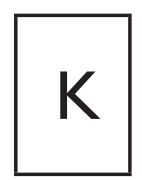

2

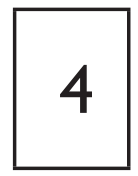

3

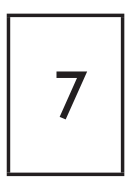

4

Après avoir précisé que, sur le recto, on peut trouver deux lettres possibles : $\mathrm{E}$ ou $\mathrm{K}$ et que, de la même manière, on peut trouver sur le verso deux chiffres possibles : 4 ou 7 , on pose la question : quelles cartes faut-il retourner pour vérifier l'affirmation suivante: si une carte a une voyelle d'un côté, elle a un chiffre pair de l'autre?

La solution est qu'il faut retourner les cartes I et 4, mais l'immense majorité des sujets de l'expérience choisit les cartes I et 3 . Ce faisant, ils se concentrent sur les cas qui confirment la règle plutôt que sur ceux qui l'infirment. En effet, il paraît naturel de considérer que la carte 3 confirme la règle que prescrit l'énoncé du problème, ce qui est le cas si l'on trouve une voyelle en examinant son verso. En réalité, on pourrait y découvrir une consonne sans que cette règle en soit violée ; la seule carte qui peut (la première mise à part) en établir la validité est la quatrième, car si elle portait à son verso une voyelle, il serait évident que l'énoncé est faux. Il existe donc une tendance à traiter une relation 
d'implication comme une relation d'équivalence. C'est le célèbre sophisme de l'affirmation du conséquent : (( $\mathrm{P} \supset \mathrm{N}) \wedge \mathrm{N}) \supset \mathrm{P}$, c'est-à-dire si $\mathrm{P}$ alors $\mathrm{N}$, or $\mathrm{N}$ donc $\mathrm{P}$ (qui est un raisonnement non valide). En toute logique (Modus Tollens) on peut dire : $\left((\mathrm{P} \supset \mathrm{N})^{\wedge} \sim \mathrm{N}\right) \supset \sim \mathrm{P}$, c'est-à-dire si $\mathrm{P}$ alors $\mathrm{N}$, or non-N donc non-P.

Ce processus mental de la confirmation a beaucoup de chances de pérenniser toutes sortes de stéréotypes (parce que ceux-ci sont souvent de la forme tous les $\mathrm{X}$ sont $\mathrm{Y}$ ). Il propose aussi un éclairage simple, mais puissant, pour comprendre la longévité des croyances. En effet, on trouve souvent le moyen d'observer des faits qui ne sont pas incompatibles avec un énoncé douteux, mais cette démonstration n'a aucune valeur si on ne tient pas compte de la proportion ou même de l'existence de ceux qui le contredisent.

Si cette appétence pour la confirmation n'est pas l'expression de la rationalité objective, elle nous facilite aussi, d'une certaine façon, l'existence. Ainsi, le processus d'infirmation est-il sans doute plus efficace si notre but est de chercher la vérité, comme le soulignait déjà Bacon, parce qu’il diminue la probabilité de chance de considérer comme vrai quelque chose de faux. En revanche, il exige un investissement en temps ${ }^{6}$ qui peut, à la limite, être considéré comme absurde, puisqu'il ne s'agit alors que de prendre une décision satisfaisante. Comme beaucoup d'auteurs l'ont fait remarquer, on pense tout particulièrement à la notion de rationalité procédurale chez le Nobel Herbert Simon $(1959,1963)^{7}$, les individus choisissent souvent plutôt que l'inférence optimale, une inférence satisfaisante. Dans le fond, les acteurs sociaux acceptent certaines explications objectivement douteuses parce qu'elles paraissent pertinentes dans le sens que Sperber et Wilson (1989) ont donné à ce terme. En situation de concurrence, expliquent-ils, on optera pour la proposition qui produit le plus d'effet cognitif possible pour le moindre effort mental. La solution objectivement bonne, lorsqu'elle existe, est souvent la plus satisfaisante, mais les individus non pas toujours assez d'imagination

6 Comme le fait remarquer Friedrich (1993).

7 Mais Mill avait déjà fait dans son Système de logique des remarques similaires. 
et de motivation pour la concevoir ${ }^{8}$ et s'abandonne fréquemment à ce que Fiske et Taylor (1984) nomment notre «avarice cognitive». Cette «avarice cognitive» conduit souvent à endosser des croyances douteuses mais relativement convaincantes parce que sur nombre de sujets, la motivation des individus n'est pas assez forte pour leur inspirer des procédures de contrôle mental. C'est que si les connaissances méthodiques produisent souvent un effet cognitif supérieur aux propositions seulement «satisfaisantes » que sont les croyances, elles impliquent aussi un coût d'investissement plus important. La probabilité de chances d'endosser celles-là sont dépendantes de la facilité à rencontrer celles-ci.

Parce que les croyances proposent souvent des solutions qui épousent les pentes naturelles de l'esprit elles produiront souvent un effet cognitif qui sera très avantageux au regard de l'effort mental impliqué.

Une fois une idée acceptée, les individus, comme le montrent par exemple Ross, Leeper et Hubbard (1975) et Ross et Leeper (1980), persévéreront généralement dans leur croyance. Ils le feront d'autant plus facilement que la diffusion accrue et non sélective de l'information rendra plus aisée et plus probable la rencontre de «données » confirmant leur croyance. Je ne crois pas du tout, comme l'affirme l'essayiste Nicholas Carr (2008), qu'Internet reprogramme biologiquement notre cerveau, en revanche, le fait qu'un esprit en quête d'informations sur Internet dépende en partie de la façon dont un moteur de recherche l'organise me paraît acceptable. Ce que révèle le Web ce n'est pas une nouvelle façon de penser, mais une façon très ancienne (biais de confirmation) au contraire.

Les choses peuvent être formalisées assez simplement. Si l'on note N le nombre d'informations qui caractérise un marché cognitif considéré et $\mathrm{P}_{\mathrm{a}}$ la probabilité qu’un énoncé considéré toutes choses égales par ailleurs soit en adéquation avec les croyances préalables d'un individu, on voit bien que $\mathrm{P}_{\mathrm{c}}$, la possibilité qu’un individu puisse recourir au biais de confirmation (c'est-à-dire qu'il rencontre

8 J'ai montré (Bronner, 2006b, 2007a et b) de façon expérimentale que, face à des énigmes où il existe une solution objective, les sujets tendent à considérer cette solution comme supérieure aux autres... à condition de parvenir à la découvrir ce qui ne survient pas le plus fréquemment. 
effectivement un énoncé qui confirme sa croyance), est une probabilité composée disjonctive ${ }^{9}$ dont on peut rendre compte par cette formule :

$$
P_{c}=1-\left[\left(1-P_{a}\right)^{N}\right]
$$

Or, puisque o $>\mathrm{P}_{\mathrm{a}}>\mathrm{I}$, Pc tend vers I quand $\mathrm{N}$ tend vers l'infini. En d'autres termes, plus N, le nombre d'informations disponibles augmente, plus la possibilité pour l'individu d’avoir recours au biais de confirmation est forte.

Quelqu'un croit-il que la CIA a fomenté les attentats du II septembre? Il trouvera grâce à n'importe quel moteur de recherche sur Internet, et en quelques instants, des centaines de pages lui permettant d'affermir sa croyance. La consultation de sources d'informations qui n'épousent pas les représentations du monde de l'individu peut aisément lui paraître comme une perte de temps ${ }^{10}$.

Si l'on tient compte de ce mécanisme de recherche sélectif de l'information, il s'ensuit que, ceteris paribus, la diffusion non-sélective de toutes sortes de données est de nature à amplifier le biais de confirmation et donc la pérennité de l'empire des croyances, ce qui constitue un paradoxe remarquable de notre contemporanéité informationnelle. Mais il y a plus, et quelque chose que ne semblent pas avoir bien vu encore les différents commentateurs de la culture Internet, c'est qu'il s'agit d'un marché cognitif hypersensible à la structuration de l'offre et, mécaniquement, à la motivation des offreurs. C'est l'un des facteurs principaux de l'organisation de la concurrence cognitive sur ce marché.

\section{MOTIVATION DES CROYANTS}

Internet, ne véhicule pas que des idées fausses ou douteuses, on peut y trouver, bien entendu toutes sortes d'informations fiables, et c'est un outil précieux. Jusqu'à présent, nous avons vu que la diffusion non sélective de l'information était favorable à l'expression du biais de confirmation, une question cruciale reste dans l'ombre: est-il possible de se faire une idée de la nature de

9 Les probabilités composées disjonctives sont des probabilités dites en «ou» par opposition aux probabilités conjonctives dites en «et».

I0 Un certain nombre de recherches en psychologie sociale attestent de ce fait: voir par exemple Roussiau et Bonardi (200I). 
la concurrence qui caractérise la coexistence sur ce marché de produits contradictoires? L'expression de ce biais de confirmation sera-t-elle encore amplifiée par la valeur de $\mathrm{P}_{\mathrm{a}}$ sur ce marché cognitif particulier qu'est Internet?

Une façon d'éclairer cette question est de se demander ce qu'un internaute, sans idée préconçue sur un sujet, risque de rencontrer comme point de vue sur Internet à propos d'un thème vecteur de croyances. Cette démarche ${ }^{11}$ tient compte des résultats d'enquête sur les pratiques culturelles des Français à l'ère numérique qui montrent, sans surprise, que nos concitoyens consultent de plus en plus fréquemment Internet et que plus de la moitié d'entre eux possèdent chez eux une connexion à haut débit. On peut remarquer encore que, dans la tranche des 15-24 ans, et pour la première fois depuis l'apparition de la télévision, le temps passé devant le petit écran a baissé alors que celui consacré à Internet ne cesse de progresser (tandis que baisse aussi le temps consacré à la lecture de journaux, de livres et à l'écoute de la radio). Cela indique ceteris paribus que la recherche d'information sur Internet représente une proportion croissante de la demande des jeunes sur le marché cognitif. Par ailleurs, la moitié des internautes, explique-t-on, passent plus de $70 \%$ de leur temps de connexion à rechercher de l'information grâce à des moteurs de recherche et, parmi ceux-ci, Google a conquis une position oligopolistique. En outre, lorsque que l'internaute échoue dans sa recherche, il tente, un second essai avec un mot-clé différent mais sur le même moteur de recherche dans 76,4\% des cas plutôt que d'essayer un autre outil. Ceci m’a incité à utiliser le moteur de recherche Google pour simuler la façon dont un internaute moyen pouvait accéder à une certaine offre cognitive sur cinq sujets que je vais détailler plus bas. Google doit une partie de sa popularité à certains dispositifs techniques simples et astucieux. Les véritables algorithmes qui organisent l'efficacité du premier moteur de recherche mondial demeurent secrets, mais l'on sait que la clé de voûte se nomme pagerank (PR). Il s'agit d'une note chiffrée qui évalue l'intérêt d'un site en fonction de sa popularité. Cette mesure est établie principalement par le nombre de liens qui pointent vers un site et le nombre de ces liens est pondéré lui-même par 
la popularité des sites desquels ils sont issus. Les spécialistes ont noté que cette mesure est logarithmique, c'est-à-dire que certains paliers atteints, il devient de plus en plus difficile de progresser. Pour établir cette évaluation, des milliers de programmes robots parcourent la toile mondiale et un site a d'autant plus de chances d'être visité qu'il a un PR élevé. Son fonctionnement même indique donc quelque chose de la disponibilité de l'offre sur Internet.

À ce titre, je me suis demandé ce que proposait Google à propos de cinq sujets : l'astrologie, le Monstre du Loch Ness, l’aspartam (qui est soupçonnée parfois d'être cancérigène), les cercles de culture (crop circles) et la psychokinèse.

Ces cinq types de propositions mont paru intéressants à tester dans la mesure où ils recouvrent des domaines de crédulité bien différents et donc un grand nombre de sites de sensibilités différentes (voir les annexes disponibles en ligne: http://www.droz.org/fr/livre/?GCOI=2600/I00723840) et permettent d'espérer éviter de rencontrer des communautés de croyants trop homogènes.

\begin{tabular}{|c|c|c|c|c|}
\hline PSYCHOKYNĖSE & $\begin{array}{c}\text { MONSTRE DU } \\
\text { LOCH NESS }\end{array}$ & ASPARTAM & $\begin{array}{c}\text { CERCLE DE } \\
\text { CULTURE }\end{array}$ & ASTROLOGIE \\
\hline $\begin{array}{c}\text { POuvoir caché } \\
\text { de l'esprit }\end{array}$ & cryptozoologie & technophobie & $\begin{array}{c}\text { croyance extra- } \\
\text { terrestre }\end{array}$ & mancie/tradition \\
\hline
\end{tabular}

L'examen auquel je me suis livré concernant ces sujets ne prétend pas trancher la question de savoir ce que l'internaute trouvera convaincant ou non parmi toutes les informations que lui proposera Google, même s'il est entendu que la nature de la source telle qu'elle est perçue par l'individu aura une influence sur la crédibilitét ${ }^{2}$ de l'information.

Ces thèmes ont été choisis aussi parce que l'orthodoxie scientifique conteste la réalité des croyances qu'ils inspirent. C'est vrai pour la psychokinèse ${ }^{13}$, le monstre de Loch Ness, les cercles de culture conçus comme des signes extraterrestres, c'est vrai encore de l'astrologie ${ }^{14}$ 'est vrai aussi des suspicions qui

12 Sur cette question classique voir, par exemple, Bovard (1953), Chaiken (1980) ou Shérif et Hovland (1961).

13 Voir de Launière (1980) ou encore Broch (1989).

14 L'astrologie, en effet, est une doctrine qu'à ma connaissance aucun scientifique ne songerait 
ont porté un temps sur l'aspartam ${ }^{15}$. Ces cinq thèmes offrent donc un poste d'observation intéressant pour évaluer le rapport de force sur ce marché cognitif entre des informations considérées comme orthodoxes par la communauté scientifique et d'autres qui ne le sont pas et que je considérerai pour cette raison, et à titre gnoséologique, comme des $\operatorname{croyances}^{16}$, non pour les disqualifier - il n'est pas besoin d'examiner la question de la vérité de ces énoncés ici -, mais pour mettre en exergue une réalité qui, sans cette distinction, se dissiperait. Pour le dire encore autrement, ce qu'il s'agira d'observer ici c'est la concurrence entre des types d'énoncés qui prétendent rendre compte des mêmes phénomènes - les uns pouvant se réclamer du consensus de l'expertise scientifique, les autres non - telle qu'elle peut être saisie par certaines des traces qu'elle laisse sur la toile. Celles-ci ne sont, en aucun cas, une expression représentative de l'opinion publique en général ou de celle des internautes en particulier bien entendu, elles donnent, en revanche, une idée de l'offre proposée par Internet à une demande d'information.

Seulement, le moteur de recherches propose souvent des centaines voire des milliers de sites à propos de n'importe quel sujet et l'on sait que l'internaute n’en consulte jamais autant pour s'informer. J’ai donc limité la recherche, pour la rendre réaliste, à ce que l'on sait du comportement de celui ou celle qui utilise un moteur de recherche : $65 \%$ se contentent de consulter la première page (les

à défendre. Non par aversion idéologique, mais simplement parce que les deux hypothèses sur lesquelles elle repose: I) notre caractère serait influencé, voire déterminé, par la configuration stellaire au moment de notre naissance - 2) notre destin, notre avenir et l'avenir du monde en général serait lui aussi influencé ou déterminé par l'évolution de ces configurations stellaires, sont invalidées par des arguments qui paraissent définitifs. Pour un état de l'art de arguments généralement retenus par la communauté scientifique, voir Lequèvre (2002).

I5 II s'agit de l'ester méthylique d'un dipeptide, une molécule constituée de l'association de deux acides aminés. C'est une substance non-nutritive qui a une saveur sucrée deux cents fois supérieure à celle du sucre. Cette suspicion tire son origine de l'existence d'une étude italienne - Soffriti, M. et al. (2006) - qui prétendait avoir montré que la consommation de ce produit pouvait favoriser l'apparition de tumeur cancéreuse chez le rat. II se trouve que cette expérimentation fut considérée comme nulle par la communauté scientifique, notamment par l'AESA (Autorité Européenne de Sécurité des Aliments), parce que les biais du protocole étaient si importants qu'ils interdisaient de prendre au sérieux les résultats obtenus: EFSA (2006).

I6 La question des rapports entre croyance et connaissance est complexe, j’ai proposé (Bronner 2003) des éléments de démarcation entre ces objets cognitifs qui ont des frontières poreuses. 
dix premiers sites), $25 \%$ s'arrêtent à la deuxième (les vingt premiers), seuls 5 à Io \% consultent ensuite la troisième page puis interrompent leur recherche. $95 \%$ et plus des internautes ne dépassent donc pas les trente premières pages.

Par ailleurs, $80 \%$ d'entre eux saisissent deux mots-clés ou moins dans leur requête.

La procédure que j’ai choisie fut toujours la même, parmi les milliers de sites mentionnés par le moteur de recherche, je n’ai retenu que ceux considérés comme pertinents par Google, c'est-à-dire ceux évitant les répétitions, et parmi eux, les trente premiers. Par ailleurs, la recherche se faisait en utilisant toujours les entrées les plus courtes et les plus neutres possibles: «Psychokinèse»; «Monstre du Loch Ness »; «Aspartam»; «Astrologie »; « cercles de culture ».

Les trente premiers sites proposés sur thème par Google étaient ensuite classés en quatre catégories possibles:

1. Les sites non pertinents: Un site fut considéré comme non pertinent, d'une part, lorsque son contenu prenait prétexte d'un thème pour aborder un objet tout à fait différent, comme ce site: «http://www.gizmodo.fr/2010/03/II/lemonstre-du-loch-ness-nest-pas-en-ecosse-mais-dans-mon-salon.htm》 ( $7^{\mathrm{e}}$ position avec l'entrée «monstre du Loch Ness») et proposant la vente de lampe en forme du monstre du Loch Ness. D’autre part, lorsque son contenu ne développait aucun argument ou point de vue concernant la croyance visée comme ce site (19 position avec l'entrée «aspartam») : «http://forums.futura-sciences. com/chimie/223689-formule-aspartam.html» proposant un forum discutant de la formule chimique de l'aspartam.

2. Les sites «neutres»: Un site fut considéré comme neutre lorsqu’il ménageait une part aux argumentations contradictoires des propositions orthodoxes de la science et de celles qui ne le sont pas, quel que soit l'environnement du site (même lorsqu'il s'agissait d'un site favorable à toutes sortes d'autres croyances) : par exemple le site «http://www.amessi.org/L-Aspartam» (11 ${ }^{\mathrm{e}}$ position avec l'entrée «Aspartam») de l'AMESSI (Alternatives Médecines Evolutives Santé et Sciences Innovantes) qui fait aussi bien la promotion de l'aromathérapie que celle de magnétiseurs mais qui propose cependant à propos de l'aspartam un article qui parait équilibré, rappelant qu'il n'existe aucune preuve scientifique de la dangerosité de l'aspartam, mais soulignant sans cesse que les suspicions sont encore vives, fut classé pour cette raison dans les articles «neutres». 
3. Les sites défavorables à la croyance: Un site fut considéré comme sceptique soit lorsqu'il développait les seuls arguments ou points de vue défavorables à la croyance, soit lorsqu'il ne ménageait un espace aux arguments défendus par les croyants que pour mieux souligner leur inanité (voir plus bas les remarques sur les types de site).

4. Les sites favorables à la croyance: Un site fut considéré comme favorable soit lorsqu'il développait les seuls arguments ou points de vue favorables à la croyance, soit lorsqu'il ne ménageait un espace aux arguments défendus par les sceptiques que pour mieux souligner leur inanité (voir plus bas les remarques sur les types de site)

\section{LES DIFFÉRENTS TYPES DE SITES}

Classer les sites dans ces différents types ne fut pas toujours aisé. Ce classement s'est fait en fonction de la nature de l'information proposée par les pages Web. On peut en distinguer cinq types, certains sites recouvrant plusieurs types.

1. Les dossiers: Ces sites présenteront la croyance et généralement une argumentation pour et / ou contre la croyance. Ils peuvent prendre plusieurs formes, des articles d'encyclopédie en ligne, des argumentaires militants, des exposés scientifiques, des cours etc. Il était difficile de se doter d’une règle stricte pour classer ces dossiers comme, par exemple, choisir de compter le nombre de lignes consacrées à telle ou telle part de l'argumentation pour en inférer la sensibilité du site (lorsque deux argumentations contradictoires étaient proposées), parce que tous les arguments ne se valent pas et que la longueur nécessitant leur exposition n'est pas nécessairement corrélée à leur robustesse. Il y a donc quelque chose d'impressionniste dans cette évaluation. Pour tempérer la tentation qui aurait pu être la mienne de surévaluer le nombre de sites «croyants», j’ai opté le plus souvent pour une interprétation en faveur du scepticisme par excès. Par exemple, l’article psychokinèse de l'encyclopédie en ligne Wikipedia ( $1^{\text {re }}$ position) «http://fr.wikipedia.org/wiki/Psychokinèse» propose un article qui paraît équilibré, présentant les thèses des parapsychologues comme celles de leurs détracteurs sceptiques. Cependant, l'ensemble de l'argumentation est plutôt favorable au scepticisme, même si la bibliographie proposée est, elle, équilibrée. Cette référence a donc été classée comme «sceptique».

2. Les forums: Je n’ai examiné que la première page des forums, celle quavait pré-selectionnée Google. Je n’ai pas pris en compte les liens proposés qui ren- 
voyaient à d'autres sites et qui pouvaient intervenir dans les échanges. Pour classer ce type de site parmi les sites neutres, favorables ou défavorables à la croyance, j’ai dénombré les points de vue exprimés et j’ai considéré qu’il fallait qu'en excluant les prises de position «neutres », l'un ou l'autre des avis représente $60 \%$ au moins des opinions exprimées (ce qui évitait de considérer qu'une page était favorable à la croyance si 4 étaient pour et 3 contre par exemple $)^{17}$ Pour évaluer la position de certaines internautes, leur ironie par exemple, je me suis servi des «smiles» qu'ils adjoignent souvent à leur message. Sur ces forums, il arrive que les internautes ne dépassent pas les seules déclarations du type «moi j’y crois» «ce sont des bêtises», mais on retrouve souvent aussi des arguments copiés-collés provenant des «dossiers » par exemple.

3. Les services : La plupart des sites de service proposent aussi des dossiers argumentés, mais certains non. Les sites de service concernent exclusivement le thème de l'astrologie, mais il se trouvait des sites abordant d'autres questions et proposant aussi des services astrologiques. Lorsque ces services n’étaient pas contrebalancés par une argumentation sceptique, je les ai classés comme sites croyants.

4. Les photos: certains sites, c'est particulièrement vrai de l'application «Googleimages » proposent des photos sans commentaires. Ces photos renvoient à des sites, mais me tenant toujours à la règle de l'investissement minimum de l'internaute, je n'ai pas cherché à suivre ces liens. Présentées de cette façon, ces photos ne constituent pas un argument en faveur ou en défaveur de la croyance, j’ai donc systématiquement classé ces applications comme neutres.

5. Les vidéos : Certains sites proposent des vidéos, c'est le cas de Youtube par exemple. Dans ce cas, de la même façon que pour les dossiers, les arguments présentés étaient évalués pour classer la vidéo comme favorable, neutre ou sceptique. Les vidéos, qu'elles soient sceptiques ou «croyantes», beaucoup plus que les dossiers écrits, présentaient des argumentations militantes et étaient plus simples à classer pour cette raison. D’autres sites proposaient un ensemble de vidéos sur le thème. C'est encore le cas d'un utilitaire Google qui propose sur une première page plusieurs vidéos sur un thème. Dans ce cas de figure, j’ai dénombré les vidéos favorables et défavorables en utilisant la règle des $60 \%$ pour classer la page. 


\section{LES RÉSULTATS PAR THÈME}

\section{La psychokinèse}

La recherche sur le thème de la psychokinèse entendu comme la capacité à influencer mentalement un objet, un processus ou un système sans l'utilisation de mécanismes ou d'énergies connue s'est faite le 20/08/ıo et le 23/08/10. Ce thème est un classique des études parapsychologiques. On aurait pu tout aussi bien convoquer celui de la télépathie par exemple, mais ce terme, d'un usage plus courant, risquait d'augmenter le nombre de sites non pertinents (le termes «psychokinèse », au contraire, a permis d'éviter de rencontrer un seul site non pertinent). Les trente premiers sites présents sur ce thème présentent essentiellement des argumentaires sous forme de dossiers, et marginalement de vidéos.

Types de sites concernant la psychokinèse ${ }^{18}$

\begin{tabular}{|c|c|c|c|c|c|}
\hline & DOSSIERS & FORUMS & SERVICE & PHOTOS & VIDÉOS \\
\hline PSYCHOKINĖSE & 23 & 7 & I & - & 5 \\
\hline
\end{tabular}

Parmi eux, les sites favorables à l'hypothèse de l'existence de pouvoirs de l'esprit arrivent en tête devant les sites neutres puis les sites défavorables. Ils représentent, si l'on ne tient compte que des sites prenant partie clairement, $74 \%$ des opinions exprimées, contre $26 \%$ pour les sceptiques.

Sensibilités des 30 premiers sites proposés par Google sur le thème de la psychokinèse

\begin{tabular}{|c|c|c|c|c|}
\hline & FAVORABLES & DÉFAVORABLES & NEUTRES & NON PERTINENTS \\
\hline PSYCHOKINĖSE & $17(74 \%)$ & $6(26 \%)$ & 7 & 0 \\
\hline
\end{tabular}

18 Certains sites présentaient à la fois des dossiers et des services sur la même page, par exemple, ils ont donc été comptés dans plusieurs catégories. Pour cette raison, le nombre d'occurrences dépasse 30. 


\section{Le monstre du Loch Ness}

La recherche menée concernant le thème du monstre du Loch Ness évoquant l'hypothèse de l'existence d'une créature inconnue de la zoologie officielle ou, au minimum, considérée par elle comme disparue, dans les eaux du lac écossais, s'est faite le i3/o7/10. On constatera que ce thème convoque un grand nombre de sites non pertinents ( $27 \%$ ) ce qui s'explique par la notoriété de cette créature légendaire. Elle inspire des fictions, des objets que certains sites relayent et qui sont sans rapport avec la croyance en elle-même. Par exemple, le site Amazon (www.amazon.fr/Scoubidou-monstre-Loch-Ness/dp/B000BKRZMA) propose une bande-dessinée du célèbre chien Scoubidou qui affronte le monstre du Loch Ness... Là aussi, ce sont les dossiers qui représentent le plus souvent la nature des pages que propose Google, même si le caractère visuel de certaines «preuves» fait arriver en deuxième position les sites proposant des vidéos.

Type de sites concernant le monstre du Loch Ness

\begin{tabular}{|c|c|c|c|c|c|}
\hline & DOSSIERS & FORUMS & SERVICE & PHOTOS & VIDÉOS \\
\hline MONSTRE DU LOCH NESS & 17 & 1 & 1 & 1 & 6 \\
\hline
\end{tabular}

Ce sont les sites défendant la croyance qui arrivent en première position devant les sites non pertinents. Ils représentent, si l'on ne tient compte que des sites prenant partie clairement, $78 \%$ des opinions exprimées, contre $22 \%$ pour les sceptiques.

Sensibilités des 30 premiers sites proposés par Google sur le thème du monstre du Loch Ness

\begin{tabular}{|c|c|c|c|c|}
\hline & FAVORAbles & DÉfaVorables & NeUtres & NON PERTinents \\
\hline Monstre du Loch NesS & $14(78 \%)$ & $4(22 \%)$ & 4 & 8 \\
\hline
\end{tabular}




\section{Aspartam}

La recherche menée concernant le thème de l'aspartam évoquant l'hypothèse, contre l'expertise scientifique internationale, d'une dangerosité de cette molécule, s'est faite le I5/o7/ıo. J’ai choisi le mot clé «aspartam» qui me paraissait moins biaisant qu'«aspartam et santé» ou «aspartam et cancer». L'inconvénient d'un énoncé aussi vague est qu'il était moins discriminant, on retrouve donc aussi pour ce thème un nombre non négligeable de sites «non pertinents» $(23 \%)$. L'image n'étant pas une ressource très percutante pour ce thème, les arguments sont développés essentiellement sous la forme de dossiers et un peu de forums (on retrouve une seule vidéo qui est un extrait de l'émission $C$ dans l'air).

Type de sites concernant la dangerosité de l'aspartam

\begin{tabular}{|c|c|c|c|c|c|}
\hline & DOSSIERS & FORUMS & SERVICE & PHOTOS & VIDÉOS \\
\hline ASPARTAM & 16 & 5 & 2 & - & 1 \\
\hline
\end{tabular}

Ce sont les sites défendant la croyance qui arrivent, une fois de plus, en première position devant les sites non pertinents. Ils représentent, si l'on ne tient compte que des sites prenant partie clairement, 70\% des opinions exprimées, contre $30 \%$ pour les sceptiques, ce qui est le plus faible rapport de force recueilli. Compte tenu de l'étroitesse de l'échantillon considéré, il convient de rester prudent, mais peut-être est-ce parce que des enjeux économiques existent sur ce thème (au moins pour les producteurs d'aspartam) et que, dans ces conditions, certains sceptiques peuvent avoir des motivations plus fortes d'exprimer leur point de vue, nous y reviendrons.

Sensibilités des 30 premiers sites proposés par Google sur le thème de la dangerosité de l'aspartam

\begin{tabular}{|c|c|c|c|c|}
\hline & FAVORABLES & DÉFAVORABLES & NEUTRES & NON PERTINENTS \\
\hline AsPARTAM & $14(70 \%)$ & $6(30 \%)$ & 3 & 7 \\
\hline
\end{tabular}




\section{Les cercles de culture}

La recherche menée concernant le thème des cercles de culture s'est faite du 19 au 21/07/20Io. Les cercle de culture encore nommés crop circles sont de grands cercles qui apparaissent mystérieusement, généralement dans des champs de blé. Ceux-ci peuvent être de simples cercles ou dessiner des figures plus complexes. Personne ne doute de la matérialité de ces phénomènes. Cependant, il en existe plusieurs interprétations concurrentes. La plus immédiate est celle du canular, d’autant que ces phénomènes, étant principalement apparus dans le Sud de l’Angleterre dans les années 1980, pouvaient facilement être reproduits artificiellement. D’ailleurs, en septembre i99ı, deux artistes, Doug Bower et Dave Chorley prétendirent qu'ils étaient les auteurs de plus de deux cents crops circles depuis 1976. Un peu partout dans le monde, des groupes appelés des circle makers revendiquent la création de ces «œuvre d'art». Ceci n’empêche pas certains de prétendre que ces cercles ne peuvent pas tous être des canulars car, disent-ils, certains paraissent trop complexes, trop réguliers pour être réalisés de main d'hommes en une seule nuit. Ils évoquent encore des phénomènes biochimiques se produisant au contact de ces cercles qui excluent l'hypothèse du canular ou de l'œuvre d'art. Ceux-ci évoquent alors le plus souvent l'idée de l'action d'une technologie extra-terrestre. La théorie de pistes d'atterrissage pour des extra-terrestres est une des plus courantes et a été popularisée par le film Signes de Night Shyamalan.

Là encore, ce sont les dossiers qui se taillent la part du lion parmi les types de sites qui se saisissent de ce sujet, mais on notera, ce qui n'est pas une surprise compte tenu de la nature visuel de cercles de culture, la présence notable de sites fondés essentiellement sur l'image.

Type de sites concernant les cercles de culture

\begin{tabular}{|c|c|c|c|c|c|}
\hline & DOSSIERS & FORUMS & SERVICE & PHOTOS & VIDÉOS \\
\hline CERCLE DE CULtURE & 19 & 8 & 1 & 1 & 7 \\
\hline
\end{tabular}


Ce sont les sites défendant la croyance qui arrivent en première position. Ils représentent, si l'on ne tient compte que des sites prenant partie clairement, $87 \%$ des opinions exprimées, contre i $3 \%$ pour les sceptiques. On notera cependant que ce thème recueille le plus grand nombre de sites «neutres». Même lorsque les dossiers sont proposés par des sites favorables à toutes sortes de croyances, il arrive fréquemment qu'ils expriment un avis mitigé et prudent, un peu comme pour le thèse de la psychokinèse, peut-être parce que, comme lui, il a donné lieu à des supercheries reconnues.

Sensibilités des 30 premiers sites proposés par Google sur le thème des cercles de culture

\begin{tabular}{|c|c|c|c|c|}
\hline & favorables & Défavorables & NeUtres & NON Pertinents \\
\hline Cercle de Culture & $14(87 \%)$ & $2(13 \%)$ & 12 & 2 \\
\hline
\end{tabular}

\section{L'astrologie}

La recherche menée concernant le thème de l'astrologie s'est faite le I2/07/Io. Si les dossiers sont, une fois de plus bien représentés pour ce thème, la présence importante de la dimension des services proposés confère à ce thème un statut un peu particulier. Le plus souvent ces sites de services s'accompagnent d'argumentaires en faveur de l'astrologie, de cours ou de témoignages faisant preuve de l'efficacité de ce technique prétendant prédire l'avenir et établir la personnalité des individus, mais leur dimension marchande est presque toujours évidente.

Type de sites concernant l'astrologie

\begin{tabular}{|c|c|c|c|c|c|}
\hline & DOSSIERS & FORUMS & SERVICE & PHOTOS & VIDÉOS \\
\hline ASTROLOGIE & 20 & 2 & 24 & - & - \\
\hline
\end{tabular}

Les enjeux économiques autours de ce thème expliquent sans doute la rapport de force très favorable à la croyance qu'on observe parmi les 30 premiers sites proposés par le moteur de recherche. 
Sensibilités des 30 premiers sites proposés par Google sur le thème de l'astrologie

\begin{tabular}{|c|c|c|c|c|}
\hline & FAVORABles & DÉFAVORABLES & NEUTRES & NON PERTINENTS \\
\hline Astrologie & $28(97 \%)$ & $1(3 \%)$ & - & 1 \\
\hline
\end{tabular}

Le facteur économique qui n’est pas à négliger n’explique cependant pas le rapport de force toujours défavorable pour la connaissance orthodoxe que l'on peut observer sur les 5 thèmes évalués, même lorsque ceux-ci ne convoquent pas d'enjeux d'intérêts évidents. En fait, Internet révèle des interactions informationnelles très particulières. La structuration de l'offre, notamment, est, sur certains sujets, largement plus dépendante de la motivation des offreurs que de celles des demandeurs, et surtout, de ceux qui seraient en mesure techniquement de constituer des offres concurrentielles et contradictoires. Pour l'exprimer autrement, les croyants sont généralement plus motivés que les noncroyants pour défendre leur point de vue et lui consacrer du temps.

Pour cette première raison que la croyance est partie prenante de l'identité du croyant, il aura facilement à cour de chercher de nouvelles informations affermissant son assentiment. Un conspirationniste, par exemple cherchera à retenir quelques-uns des arguments qui permettent de croire que les attentats du i s septembre ont été fomentés par la CIA. Le non-croyant sera souvent dans une position d'indifférence, il refusera la croyance, mais sans avoir besoin d'une autre justification que la fragilité de l'énoncé qu'il révoque. Ce fait est d'ailleurs tangible sur les forums. Parmi les 23 forums, tout thèmes confondus qui ont été évalués, 9 ne peuvent être utilisés ici parce qu'ils relèvent de la catégorie «non-pertinent». Parmi les I4 restants, 2 I I points de vue sont exprimés, 83 défendent le point de vue de la croyance, 45 la combattent et 83 sont neutres (voir annexes en ligne: http://www.droz.org/fr/livre/?GCOI=2600। I00723840). Ce qui frappe à la lecture des forums c'est que les sceptiques se contentent souvent d'écrire des messages ironiques, ils moquent la croyance plutôt qu'ils n’argumentent contre, alors que les défenseurs de l'énoncé convoquent des arguments certes inégaux (liens, vidéos, paragraphe copié/collé...) mais étayent leur point de vue. Parmi les posts proposés par ceux qui veulent défendre la croyance, $36 \%$ 
sont soutenus par un document, un lien ou une argumentation développée, alors que ce n'est le cas que dans I0 \% des cas pour les posts de «non-croyants ».

Une seconde raison est que ceux qui seraient en mesure d'opposer des argumentaires robustes aux allégations des croyants n'ont pas beaucoup d'intérêt à le faire. Reprenons l'exemple de l'astrologie, n'importe quel astronome pourrait facilement faire la promotion de certaines des raisons qui conduisent à trouver les propositions astrologiques douteuses, certains ne s'en privent pas d'ailleurs. Mais la plupart du temps, ils ne se sentent pas plus quagacés par les allégations astrologiques, celles-ci ne représentent aucun danger pour eux, les combattre serait chronophage et sans intérêt institutionnel. Ils ont raison en un sens, mais la conséquence de leur démotivation est que les acteurs de l'astrologie, beaucoup plus résolus qu'eux, ont réussi à instaurer un oligopole cognitif paradoxal. On trouve beaucoup plus aisément leur point de vue sur Internet que celui de ceux qui pourraient facilement démontrer l'inanité des thèses astrologiques. C'est un fait qui était déjà avéré pour le marché du livre sans doute, mais qu’Internet amplifie dans la mesure où il réduit considérablement les coûts de l’offre.

L'exception à cette tendance, vient des milieux rationalistes qui, pour des raisons de militance, trouvent une motivation suffisante pour consacrer du temps et de l'énergie mentale à s'opposer. Parmi l'ensemble des points de vue contestant les cinq croyances mentionnées, on en trouve $37 \%$ qui sont émis par des sites rationalistes revendiqués (zététique, AFIS, sceptiques du Québec...). Si l'on exclut le thème de l'aspartam sur lequel les sceptiques ne se sont pas beaucoup penchés, on atteint $54 \%$.

Il me paraît acceptable de supposer que cette concurrence sur le marché cognitif puise à la source de la motivation des croyants qui est supérieure à celles des sceptiques. Sans doute, lorsqu'il existe un débat social très vif sur une question, les non-croyants sont-ils parfaitement capables de se mobiliser et occuper leur espace naturel dans le débat public, mais la plupart du temps, tout se passe comme s'il existait une sorte de ventre mou dans notre rationalisme contemporain dans lequel les croyances se taillent allégrement un espace très conséquent et un peu paradoxal. 
Le fait qu'Internet est un marché cognitif hypersensible à la structuration de l'offre et à la motivation des offreurs a une portée qui dépasse d’ailleurs le seul sujet des croyances, puisque, comme Keen (2007) l'a fait remarquer à propos du site Digg ${ }^{19}$ qui compte 900 oo० utilisateurs inscrits, 30 personnes ont suffi à déterminer $\mathrm{I} / 3$ des textes présents sur la page d'accueil. Sur Netscape ${ }^{20}$, un seul utilisateur était derrière 2 I7 articles publiés soit I3 \% de tous les articles figurant dans la liste des articles les plus appréciés pendant la période.

\section{CONCLUSION}

Il y a donc une tendance corrélative entre la facilité d'accession à l'information et la massification de sa diffusion et celle des croyances. C'est un point que paraissent ne pas avoir sérieusement envisagé les promoteurs de la réduction de la «fracture numérique». Et même s'ils ajoutent à leurs vœux la volonté de réduire la «fracture cognitive» en espérant voir partout augmenter le niveau d'étude parce qu’il «implique la maîtrise de certaines compétences cognitives, critiques et théoriques, dont le développement est précisément l'objet des sociétés du savoir » (UNESCO, p. 47), ils seraient bien inspirés de ne pas attendre trop de choses de l'augmentation généralisée du niveau d'éducation des populations. Il a été montré maintes fois, en effet, qu'une bonne formation intellectuelle n’immunisait pas du tout contre l'adhésion à toutes sortes de croyances. C’est même le contraire qui est vrai si l'on en croit, par exemple, les enquêtes menées par Boy et Michelat (1986) sur les croyances des Français concernant les parasciences: «À l'évidence, [écrivent-ils (p. I85)], il faut abandonner un modèle linéaire selon lequel la proximité au rationalisme ou au mode de pensée scientifique irait de pair avec l'élévation du niveau d'études. » Ainsi, d’après leur étude, la croyance au paranormal ou à l'astrologie touche, dans l'ordre croissant: le primaire, le primaire supérieur, le secondaire et le supérieur non scientifique. Dans le même ordre d'idées, on pourrait mentionner que ceux qui adhèrent le plus facilement

19 Digg est un site participatif où les internautes sélectionnent leurs articles et sites préférés.

20 Netscape était un site d'actualité avant qu'il ne soit remplacé par AOL. 
au mythe du monstre du loch Ness ${ }^{21}$ sont, là aussi, des individus diplômés, de même pour les adeptes de l'homéopathie, comme le fait remarquer Bouchayer (1986)... On pourrait multiplier les exemples, mais les études les plus frappantes sont celles qui montrent que même les croyances extrêmes, en tout cas celles qui inspirent des actes meurtriers, paraissent plus facilement endossées par des individus ayant un niveau de diplôme supérieur à la moyenne ${ }^{22}$, il n'en va pas différemment de ceux qui adhèrent à des mouvements sectaires ${ }^{23}$.

Qu'on ne déduise pas de ces quelques lignes l'idée que je juge inutiles les efforts d'éducation ou plus généralement, les espoirs légitimes que peut susciter l'idée d'une société de la connaissance, je me borne, en tant que sociologue, à décrire certains des facteurs à l'œuvre dans la diffusion des croyances et, à ce titre, juge fascinant les paradoxes de notre contemporanéité informationnelle et les espoirs à tendance idéologique qu'elle suscite.

\section{BIBLIOGRAPHIE}

AJZEN I. et KRUGLANSKI A., 1983, «Bias and error in human judgement», European Journal of Social Psychology, 13, p. I-49.

AUTRET M., 2002, «La brouillotique nous gagne», Écrire et éditer, n³9.

BACON F., 1986, Novum Organum, Paris, PUF.

BAUERLEIN M., 2008, The dumbest generation, Londres, Tarcher / Penguin.

BINDÉ J., 2005, Vers les sociétés du savoir, Rapport Mondial de l'Unesco, éditions UNESCO.

BOUCHAYER F., 1986, «Les usagers des médecines alternatives: itinéraires thérapeutiques, culturels, existentiels», Revue française des affaires sociales, numéro hors-série, avril, p. I05-II5.

21 Selon une étude menée par Grimshaw et Lester citée par J-B. Renard (2010).

22 Voir Sageman (2004), Etienne (2005), Cherkaoui (2007), Krueger (2007) ou encore Ruby (2002).

23 Voir Duval (2002) ou Stupple (1984). 
BOVARD E. W., 1953, «Conformity to social norms in stable an temporary groups », Science, p. 361-363.

BOY D. et MICHELAT G., 1986, «Croyances aux parasciences: dimensions sociales et culturelles», La Revue française de sociologie, XXVII.

BROCH H., 1989, Le Paranormal, Paris, Seuil.

BRONNER G., 20II, The Future of Collective Beliefs, Oxford, Bardwell Press.

-, 2007, «La résistance au darwinisme: croyances et raisonnements», La Revue Française de Sociologie, p. 48-3, p. 587-607.

-, 2006a, Vie et mort des croyances collectives, Paris, Hermann.

-, 2006b, «Une théorie de la naissance des rumeurs», Diogène, 213, p. 107-132.

-, 2003, L'Empire des croyances, Paris, PUF.

CARR N., 2008, The Big Switch: Rewiring the World, from Edison to Google, New York, W. W. Norton \& Company.

CASTELLS M., 1998, L’Ère de l'information: la société en réseaux, Paris, Fayard.

CHAIKEN S., 1980, «Heuristic versus Systematic Information Processing and the Use of Source versus Message Cue in Persuasion», Journal of Personality and Social Psychology, 39, 5, p. 752-766.

CHERKAOUI M., 2007, Good intentions - Max Weber and the Paradox of Unintended Consequences, Oxford, Bardwell Press.

DONNAT O., 2008, Pratiques culturelles des Français à l'ère numérique, Paris, La Découverte/Ministère de la Culture et de la Communication.

DUVAL M., 2002, Un ethnologue au Mandarom, Paris, PUF.

DRUCKER P., 1969, The Age of Discontinuity. Guidelines to our Changing Society, New York, Harper \& Row.

EFSA: European Food Safety Authority, 2006, «Opinion of the scientific panel on food additives, flavourings, processing aids and materials in contact with food (AFC) on a request from the Commission related to a new long term carcinogenecity study of aspartame», The EFSA Journal, 356, p. I-44.

ETIENNE B., 2005, Les Combattants suicidaires, Paris, L'aube. 
FISKE S. T. et TAYLOR S. E., 1984, Social cognition, New York, Random House.

FRIEDRICH J., 1993, «Primary Detection and Minimization strategies in social cognition: a reinterpretation of confirmation bias phenomena», Psychological Review, 100, 2, p. 298-319.

GASCHKE S., 2009, Klick. Strategien gegen die digitale verdummung, Fribourg, Herder. KEEN A., 2007, The Cult of the amateur, New York, Bantam.

KRUEGER A., 2007, What Makes a Terrorist: Economics and the Roots of Terrorism, Princeton University Press.

LAUNIÈRE de C., 1980, «Aux yeux de la science officielle, la parapsychologie n'a pas encore fait ses preuves », Québec Science, I, vol.I9.

LEQUÈVRE F., 2002, «L'astrologie» in Guide critique de l'extraordinaire, éd. Mahric, Bordeaux, Les arts libéraux.

MANSELL R. et WEHN U., 1998, Knowledge Societies: Information Technology for Sustainable Development, United Nations Commission on Science and Technology for Development, New York, Oxford University Press.

MILL J. S., 1988, Système de logique, Bruxelles, Mardaga.

RENARD J-B., 2010, «Croyances fantastiques et rationalité», L'Année Sociologique, 60-I, p. $115-135$.

ROSS L. et LEEPER M. R., 1980, «The perseverance of beliefs: Empirical and normative considerations » in News Directions for Methodology of Behavioral Science: Faillible Judgement in Behavioral Research, eds Shweder et Fiske, San Francisco, Jossey-Bass.

-, et HUBBARD M., 1975, «Perseverance in self-perception and social perception: Biased attributional processes in the debriefing paradigm. », Journal of Personality and Social Psychology, 32, p. 880-892.

ROUSSIAU N. et BONARDI C., 200I, Les Représentations sociales, Hayen, Mardaga.

RUBY C. L., 2002, «Are terrorists mentally deranged?», Analysis of Social Issues and Public Policy, 2, p. 15-26. 
SAGEMAN M., 2004, Understanding Terror Networks, Philadelphia, University of Pennsylvania Press.

SHÉRIF M. et HOVLAND C. I., 1961, Social judgment, Yales, New Haven, University Press.

SIMON H., 1959, «Theories and Decision-Making in Economics and Behavioral Science», American Economic Review, 49, 3, 253-283.

-, 1963, «Economics and Psychology» in Psychology: A Study of Science, eds Koch, New York, McGraw Hill, t.Vl.

SOFFRITI M. et al., 2006, «First experimental demonstration of the multipotential carcinogenic effects of aspartame administered in the feed to Sprague-Dawley rats», Env. Health Perspect, II4, p. 379-385.

SPERBER D. et WILSON D., 1989, La Pertinence - communication et cognition, Paris, Editions de Minuit.

STEHR N., 1994, Knowledge Societies: The Transformation of Labour, Property and Knowledge in Contemporary Society, Londres, Sage.

STUPPLE D., 1984, «Mahatmas and Space Brothers: The Ideologies of Alleged Contact With Extraterrestrials», Journal of American Culture, no 7, p. I31-139.

TAPSCOTT D., 2008, Grown up digital, New York, MacGraw-Hill.

WASON P.C., 1977, «Self-contradiction», in Thinking: Reading in cognitive science, eds Johnson-Laird et Wason, Cambridge, Cambridge University Press. 